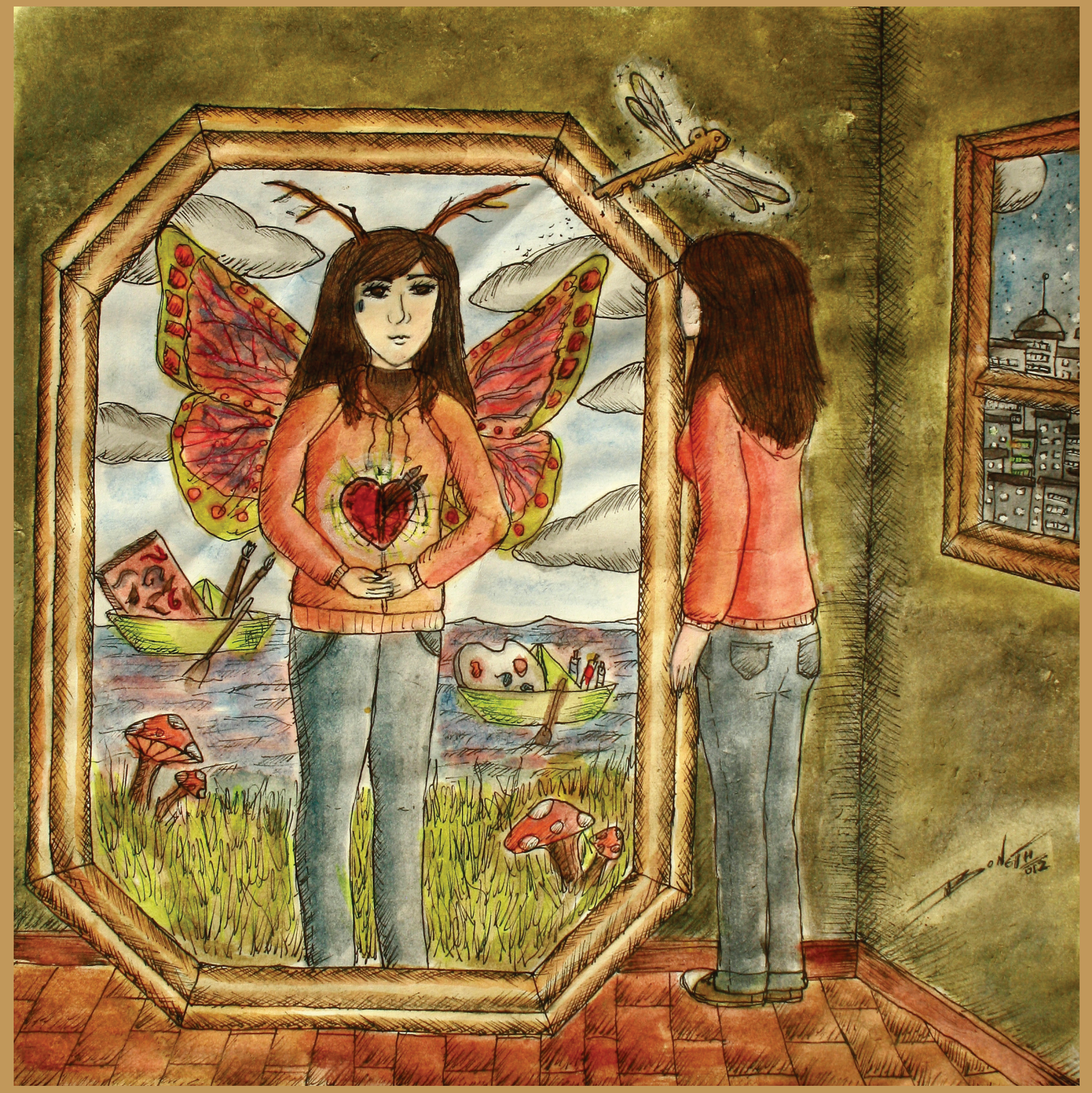

Lorna Boneth Melo - Arte sin Fronteras - Programa Talentos Especiales

German Augusto Baquero Sastre Fisioterapeuta

M. Sc. en Ciencias de la Salud con Área de Concentración en Epidemiologia Docente - Investigador del Programa de Fisioterapia de la Universidad Manuela Beltrán. Líder del Grupo de Investigación en Neurorehabilitacion Avenida Circunvalar Número 6o - oo Bogotá gbaquer@yahoo.es 


\title{
PREVALENCIA DE LA ENFERMEDAD DE ALZHEIMER ENTRE LAS ENTIDADES NEUROLÓGICAS ATENDIDAS EN FISIOTERAPIA
}

\section{Prevalence of alzheimer's disease in the neulogical diseases served in physical therapy}

Fecha de recepción: 26 de junio de 2014 - Fecha de aprobación: 1 de diciembre de 2014

\author{
RESUMEN
}

El envejecimiento de la población y probables factores ligados a sus estilos de vida parecen ser determinantes en la presentación de la Enfermedad de Alzheimer que viene teniendo una tendencia de crecimiento en la frecuencia de ocurrencia en la población que resulta afectada no solo en sus desempeños cognitivos sino en condiciones de movimiento que conjuntamente van a limitar la calidad de vida de las personas afectadas por la entidad. Objetivo. Conocer la prevalencia de la enfermedad de Alzheimer entre las entidades neurológicas atendidas en Fisioterapia. Método. Se desarrolló un estudio transversal con los pacientes mayores de 15 años atendidos por causa de afecciones neurológicas en los servicios de Fisioterapia donde se daban Practicas del Programa de Fisioterapia de la Universidad Manuela Beltrán entre febrero y noviembre de 2012, calculando la prevalencia y ocurrencia de la enfermedad de Alzheimer por género. Resultados. Durante el periodo de tiempo del estudio se atendieron un total 1062 personas a causa de enfermedades neurológicas, $19.67 \%$ de estas personas registraron como motivo de remisión a Fisioterapia la Enfermedad de Alzheimer ( $\mathrm{n}=209$, Error Estándar=0.02), correspondiendo un $77.51 \%$ de las personas con esta patología al genero femenino ( $\mathrm{n}=162$, Error Estándar=0.01) Conclusiones La enfermedad de Alzheimer tiene una prevalencia importante dentro de las entidades neurológicas, y muestra un predominio en su presentación en las mujeres.

PALABRAS CLAVE

Alzheimer, Neurología, Fisioterapia, Enfermedades Crónico Degenerativas, Demencias, Adulto Mayor

\begin{abstract}
The aging of the population and probable factors related to their lifestyles seem to be decisive in the presentation of Alzheimer's disease that has taken an upward trend in the frequency of occurrence in the population that is affected not only in their cognitive performance but able to move that joint will limit the quality of life of people affected by the entity Objective To know the prevalence of Alzheimer disease between the neurological diseases served in the Physical Therapy Services Methodology developed a cross-sectional study of patients older than 15 years treated because of neurological physiotherapy services in which they practiced the Physiotherapy Program Manuela Beltran University between February and November 2012, calculating the prevalence and occurrence of Alzheimer's disease by gender Results During the study period a total 1062 were met people because of neurological diseases, $19.67 \%$ of these people recorded as a reason for referral to Physical Therapy Alzheimer's Disease $(n=209$, Standard Error $=0.02)$, corresponding $77.51 \%$ of people with this condition the female gender ( $\mathrm{n}=162$, Standard Error = 0.01 ) Conclusions the disease Alzheimer's is a significant prevalence in neurological entities, and is predominantly in its presentation in women.
\end{abstract}

KEYWORDS

Alzheimer, Neurology, Physiotherapy, Chronic Degenerative Disease, Dementias, Elderly People 
Los cambios demográficos y de los estilos de vida de la población principalmente en sus hábitos nutricionales serian factores que conjuntamente entrarían a explicar que se esté dando una tendencia de incremento de la frecuencia de ocurrencia de casos de la Enfermedad de Alzheimer, que por sus afecciones cognitivas y conductuales va deteriorando progresivamente las capacidades de relación y participación del individuo coherente y asertivamente dentro de la realidad, reduciendo sus ejecuciones motoras en las que va a requerir cada vez mas de asistencia y cuidados especiales hasta llegar a quedar confinado en el decúbito (Loeser, 2010; van Wijngaarden y Franklin, 2013; Jagust, 2013; Kern y Behl, 2009; Thal, Tredici y Braak, 2004; Gillette-Guyonnet, Secher y Vellas, 2013; Solfrizzi, Panza, et al., 2011; Solfrizzi, Frizardi, et al., 2011; Reitz, 2013; Harrison, 2013; Head, 2013; Roman y Pascual, 2012; Boxer et al., 2013; Keating y Gaudet, 2012; Letts et al., 2011; Uzun, Kozumplik y Folnegovic-Smalc 2011)

Teniendo en cuenta las situaciones que hoy favorecen el incremento en la prevalencia de casos de la enfermedad de Alzheimer y sus trascendentales impactos en las sociedades modernas se hace necesario poder identificar la frecuencia y características con las que puede estar ocurriendo la entidad entre personas atendidas en servicios de Fisioterapia a causa de afecciones neurológicas, de tal manera que se configure un panorama que sirva de fuente argumental para planificar acciones que tiendan a fortalecer los procesos de atención haciéndolos mas eficientes y eficaces dentro de las características de la enfermedad

\section{MÉTODO}

Se desarrolló una investigación de tipo no experimental, observacional, descriptiva de corte transversal con fuentes secundarias de información (Hernández, Garrido-Latorre y Lopez-Moreno, 2000; Hernández y Velasco-Mondragón, 2000).

Hicieron parte del estudio las personas mayores de 15 años que fueron atendidas en los servicios de Fisioterapia donde se daban prácticas de la Universidad Manuela Beltrán a causa de afecciones neurológicas, y principalmente entre ellas por casos de enfermedad de Alzheimer entre febrero y noviembre de 2012.

No se aplicaron criterios de exclusión por condición de género, estado de la patología, el hecho que además de afecciones neurológicas y concretamente de casos de enfermedad de Alzheimer se dieran otras situaciones de afección del movimiento humano, o que se hubieran tenido antecedentes de atención previa en Fisioterapia por la enfermedad de Alzheimer.

La manera de selección de la población correspondió a un censo de las personas que cumplían las condiciones especificadas y que fueron atendidos en los servicios de Fisioterapia donde se dan prácticas de la Universidad Manuela Beltrán entre febrero y noviembre del año 2012.

Para la recolección de la información se emplearon los registros estadísticos de atención de pacientes de los servicios de Fisioterapia en los que estaban las Practicas de la Universidad Manuela Beltrán

En el momento la etiología de la Enfermedad de Alzheimer continua sin aclararse, pero se relaciona fuertemente con el proceso de envejecimiento adquiriendo por sus características la pertenencia a y la principal carácter cronicodegenerativo del sistema nervioso, bral. Sin embargo producto de investigaciones se ha determinado que además del proceso de envejecimiento se liga a la ocurrencia de la entidad el hecho de haber consumido dietas ricas en grasas en épocas anteriores de la vida, situación que en las sociedades modernas es un patrón nutricional altamente frecuente desde la infancia, pasando por la adolescencia y el adulto joven (Gillette-Guyonnet, Secher y Vellas, 2013; Solfrizzi, Panza, et al., 2011; Solfrizzi, Frizardi, et al., 2011; Reitz, 2013) 
de los cuales se tomó el diagnóstico de atención, el género del paciente y su edad.

Dentro del proceso de la investigación se tuvieron en cuenta las situaciones éticas de mantener la confidencialidad de la información de los pacientes encontrada en los registros estadísticos de atención consultados en el periodo del estudio, destinarla exclusivamente a los propósitos de la investigación, sin generar factores que pudieran dar lugar al reconocimiento individual de los participantes. La custodia de la información con las características descritas en su preservación y manejo fue exclusiva del investigador que desarrolló el trabajo imperando sobre él la obligación del secreto profesional.

En el análisis de resultados se emplearon medidas epidemiológicas de frecuencia, fundamentalmente prevalencias crudas y específicas por condición de género a las que en cada caso se les calculó su correspondiente nivel de error estándar. Para evaluar la relación del género con la ocurrencia de la enfermedad de Alzheimer se desarrolló una prueba de Chi Cuadrada con un grado de libertad, $\mathrm{p}<$ de 0.05 , empleándose para estos propósitos una hoja electrónica de cálculo en Excel.

\section{RESULTADOS}

Durante el periodo de febrero a noviembre de 2012 se atendieron un total de 4122 personas mayores de 15 años en los servicios de Fisioterapia donde se daban prácticas de la Universidad Manuela Beltrán por diversas situaciones que comprometían sus condiciones de movimiento, entre ellas un $25.76 \%$ ( $n=1062$, Error Estándar=0.00) registraron como motivo de intervención Fisioterapéutica afecciones que comprometían al Sistema Nervioso de diversa manera.

Entre las personas que fueron atendidas a causa de afecciones neurológicas la enfermedad de Alzheimer registró una frecuencia de $19.67 \%$ (n=209, Error Estándar=0.02) (Tabla 1)

$77.51 \%$ (n=162, Error Estándar=0.01) de las personas afectadas por la enfermedad de Alzheimer eran de género femenino $\left(\mathrm{X}_{2}=49.76, \mathrm{p}<\right.$ de 0.05 , R.M.=3.11, I.C. $95 \% 2.27-4.22$ )

29.66\% ( $n=315$, Error Estándar=0.02) de las personas que tenían afecciones neurológicas y que por ellas habían recibido atención en Fisioterapia tuvieron problemas de carácter cronicodegenerativo, dentro de los cuales un 66.34\% (n=209, Error Estándar=0.01) fueron casos de la Enfermedad de Alzheimer que con estas condiciones se convirtió en la primera entidad de carácter cronicodegenerativo del sistema nervioso, siendo seguida por la enfermedad de Parkinson que entre las de carácter cronicodegenerativo del sistema nervioso representó un 20.63\% de los casos vistos en las acciones asistenciales cumplidas en los servicios de Fisioterapia ( $n=65$, Error Estándar= o.04) (Tabla 2)

\section{DISCUSIÓN}

Las sociedades modernas han experimentado sensibles transformaciones en su composición por progresos económicos y avances científicos, junto a variaciones sociales y culturales que han llevado
Tabla 1. Prevalencia de afecciones neurológicas atendidas en servicios de Fisioterapia entre febrero y noviembre de 2012.

\begin{tabular}{|l|c|c|c|}
\hline \multicolumn{1}{|c|}{ Patología } & $\begin{array}{c}\text { Frecuencia } \\
\text { Absoluta }\end{array}$ & Prevalencia & $\begin{array}{c}\text { Error } \\
\text { Estándar }\end{array}$ \\
\hline Secuelas de ECV & 408 & $38.41 \%$ & 0.01 \\
\hline Demencia de Alzheimer & 209 & $19.67 \%$ & 0.02 \\
\hline Trauma Craneoencefálico & 85 & $8.00 \%$ & 0.02 \\
\hline Trauma Raquimedular & 80 & $7.53 \%$ & 0.02 \\
\hline Parkinson & 65 & $6.12 \%$ & 0.02 \\
\hline Polineuropatias Inflamatorias & 42 & $3.95 \%$ & 0.02 \\
\hline Traumatismos Nervio Periférico & 26 & $2.44 \%$ & 0.02 \\
\hline Trastornos Vestibulares & 23 & $2.16 \%$ & 0.02 \\
\hline Parálisis Facial & 21 & $1.97 \%$ & 0.01 \\
\hline Poliomielitis & 16 & $1.50 \%$ & 0.02 \\
\hline Distrofias Musculares & 15 & $1.41 \%$ & 0.02 \\
\hline Tumores Sistema Nervioso Central & 10 & $094 \%$ & N.E. \\
\hline Trastornos Plexo Braquial & 8 & $0.75 \%$ & N.E. \\
\hline Guillain Barre & 8 & $0.75 \%$ & N.E. \\
\hline Esclerosis Lateral Amiotrofica & 7 & $0.65 \%$ & N.E. \\
\hline Esclerosis Múltiple & 7 & $0.65 \%$ & N.E. \\
\hline Epilepsia & 6 & $0.56 \%$ & N.E. \\
\hline Encefalopatía Isquémica Hipoxica & 6 & $0.56 \%$ & N.E. \\
\hline Mielopatia Cervical & 4 & $0.37 \%$ & N.E. \\
\hline Hematoma Epidural & 4 & $0.37 \%$ & N.E. \\
\hline Radiculopatia Lumbar & 3 & $0.28 \%$ & N.E. \\
\hline Otras Afecciones & 9 & $0.28 \%$ & N.E. \\
\hline TOTAL & 1062 & & \\
\hline
\end{tabular}

Tabla 2. Enfermedades cronicodegenerativas del sistema nervioso atendidas en servicios de Fisioterapia entre febrero y noviembre de 2012.

\begin{tabular}{|c|c|c|c|c|c|}
\hline Patología & 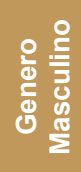 & 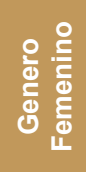 & 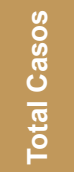 & 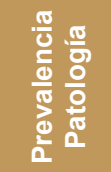 & 흔 $\frac{\frac{\hbar}{0}}{\frac{\pi}{50}}$ \\
\hline Demencia de Alzheimer & 47 & 162 & 209 & $66.34 \%$ & 0.01 \\
\hline Parkinson & 34 & 31 & 65 & $20.63 \%$ & 0.04 \\
\hline Distrofias Musculares & 10 & 5 & 15 & $4.76 \%$ & 0.04 \\
\hline Tumores Sistema Nervioso & 10 & 6 & 4 & $3.17 \%$ & 0.05 \\
\hline Esclerosis Lateral Amiotrofica & 5 & 2 & 7 & $2.22 \%$ & 0.05 \\
\hline Esclerosis Múltiple & 0 & 7 & 7 & $2.22 \%$ & 0.05 \\
\hline Ataxia Friedreich & 0 & 2 & 2 & $0.63 \%$ & N.E. \\
\hline
\end{tabular}

N.E. error estándar no calculable

a nuevas formas de comportamiento y expectativas frente al desarrollo vital de las personas como lo anota Unger (2012) y Manderson y Naemiratch (2010), panorama dentro del cual el envejecimiento de la población se ha tornado en una realidad contundente a nivel global como lo señala Arai et al. (2012), quienes anotan que es evi-

REV. COL. REH 2014 || Volumen 13 || Páginas 80 - 86 || 
dente el crecimiento de la población adulta mayor en los últimos cincuenta años pasando según ellos de representar un 5.7\% en 1960 a un $23.1 \%$ de la composición de la población en el año 2010.

Es de reconocer como lo expresa Arai et al. (2012) que es parte de las características del envejecimiento, que en la medida que ocurre este proceso se incrementa el riesgo de ocurrencia de enfermedades cronicodegenerativas por lo cual se deben procurar mejores estilos de vida en la población adolecente y adulta joven, para prevenir ciertas situaciones de salud, y sin duda preparar las respuestas asistenciales que estarán cada vez más en incremento ante las necesidades de la población que envejece.

Todos los tejidos y sistemas del organismo en la medida que pasa el proceso de vida envejecen y estas transformaciones van a generar riesgos de lesiones cronicodegenerativas, así como lo cita Loeser (2010), el envejecimiento en el sistema musculoesqueletico con reducción de la movilidad, la flexibilidad y la fuerza potencia llevan a osteoartrosis que es según este autor la enfermedad cronicodegenerativa mas frecuente en los adultos mayores.

A partir de lo anterior debe señalarse que los cambios que genera el envejecimiento en el sistema nervioso tienen que ver con menor agudeza de receptores sensoriales lo que lentifica la información aferente para los procesos de percepción y elaboración de respuestas que generen conductas y desempeños motores en el individuo, se varían los niveles de neurotransmisores como la dopamina o el acetilcolina que reduce las posibilidades sinápticas lo que es un punto que se relaciona con los hechos de la enfermedad de Alzheimer, y en la corteza cerebral se da una reducción del número de unidades neuronales que afecta los procesos sinápticos y lleva a perdida de aprendizajes y funciones adquiridas como lo señala Kern y Behl (2009), por lo que en las personas con Alzheimer tendrán como uno de los hechos principales en la clínica, cambios en la memoria por la ubicación del proceso degenerativo cortical.

Si bien es cierto que la enfermedad de Alzheimer es una entidad idiopática, cronicodegenerativa, vinculada a las situaciones del proceso de envejecimiento sobre el sistema nervioso y fundamentalmente sobre la corteza, es de resaltar que en sus orígenes esta entidad tiene una vinculación con elementos de dietas ricas en grasas y alteraciones de lípidos, donde precisamente Uzun et al. (2011) llaman la atención que para la prevención de casos de Alzheimer se debe tener control de los niveles de colesterol, pues si bien es cierto que por los cambios demográficos continuados en las sociedades modernas seria esperable en el corto y mediano plazo un inevitable incremento de casos este puede ser menor si se toma conciencia de cambiar hábitos nutricionales en población joven evitando que este sea un hecho que interactúe con el proceso de envejecimiento impactando en el número de casos de la entidad pautas que también son señaladas por Gillette-Guyonnet, Secher y Vellas (2013).

Adquirir conciencia y acciones desde la salud pública de controlar los factores nutricionales en la población joven que envejece con mayores niveles de esperanza de vida, debe ser importante enfocando este contexto no solo para la reducción de enfermedades cardiocerebrovasculares sino para la ocurrencia de casos de Alzheimer sobre lo cual la información a la población no lo hace evidente, líneas que también son expuestas en la misma orientación por Solfrizzi et al., (2011).

La prevalencia de ocurrencia de la enfermedad de Alzheimer establecida en este trabajo entre las entidades neurológicas atendidas en Fisioterapia resulta importante al estar en el orden del 19.67\% $(\mathrm{n}=209$, Error Estándar=0.02), y la relevancia de su presentación cobra mayores dimensiones si se tiene en cuenta que entre las entidades de carácter cronicodegenerativo que comprometen al sistema nervioso esta patología ocupo el primer lugar con un 66.34\% ( $\mathrm{n}=209$, Error Estándar=0.01) de los casos atendidos con estas características seguido por la ocurrencia de la enfermedad de Parkinson, hecho que coincide exactamente con lo descrito frente a la frecuencia de presentación de esta patología en el trabajo de Schapira (2013).

La ocurrencia del Alzheimer en la prevalencia encontrada en este trabajo debe poner en alerta la necesidad de establecer lineamientos políticos y administrativos para la adecuada atención que dentro de las características de la patología además de interdisciplinaria e integral se encamine a favorecer la calidad de vida de la persona y la familia, siendo conscientes que el incremento de casos no será algo del mediano plazo sino que ya es una realidad en países desarrollados que han experimentado todo el proceso de la transición epidemiológica en sus elementos demográficos, como también en países en vías de desarrollo a tal punto que como en este trabajo luego de la enfermedad cerebrovascular que fue la primera entidad neurológica atendida con el 38.41\% de los casos ( $n=408$, Error Estándar=0.01) apareció en frecuencia la enfermedad de Alzheimer, elementos que también son señalados por Chan et al. (2013)

Acorde a lo encontrado en las prevalencias de este trabajo es dable pensar que se afianza en la frecuencia de los motivos de atención e intervención en personas adultas mayores afectadas por entidades neurológicas tres patologías tanto en países desarrollados como en vías de desarrollo que en su orden serian, la enfermedad cerebrovascular, el Alzheimer y el Parkinson que entrarían a comprometer seriamente las posibilidades de desempeño motor y funcionalidad del adulto mayor reduciendo sus participaciones sociales lo que implicaría un compromiso de la calidad de vida y devastadores efectos económicos para los sistemas de salud y las familias que deberán atender no solo procesos continuados de rehabilitación, sino la adquisición de servicios especiales para el cuidado dentro de los ambientes domiciliarios que eviten complicaciones que aumenten la letalidad en las personas comprometidas por estas entidades.

Si la prevalencia de la enfermedad de Alzheimer logra niveles como los de este trabajo y tendencias de crecimiento sostenido como las apuntadas por Chan et al. (2013), estamos cerca de ver su ocurrencia no solo como un problema neurológico sino como un problema de salud publica al mirar las enfermedades cronicodegenerativas en las cuales podría ocupar los primeros lugares en casos de ocurrencia, luego de las musculoesqueleticas como la osteoporosis y la artrosis, lo que es concordante con lo anotado en el trabajo de Loeser (2010). 
El que se haya establecido en los resultados de este artículo que la ocurrencia de Alzheimer es mas frecuente en las mujeres con respecto a los hombres y se tenga una relación de genero con la ocurrencia de la enfermedad al punto que las mujeres tendrían 3.11 mas veces riesgo de tener la entidad frente a los hombres puede tener varias situaciones que ayudarían a explicar la tendencia partiendo inicialmente de consideraciones demográficas donde las mujeres tienen mayores niveles de esperanza de vida en comparación a los hombres lo cual haría que ellas experimentaran más los cambios del envejecimiento y por ende las posibilidades de terminar como casos de Alzheimer, pero también biológicos si se tiene en cuenta lo dicho por Vest y Pike (2013), quien anota que la reducción de estrógenos en la mujer favorecería cambios metabólicos en niveles de grasas que ayudarían a la aparición del Alzheimer, siendo de resaltar que este autor también anota que en el hombre la reducción de andrógenos llevaría a facilitar la ocurrencia de casos de la patología.

Luego de las consideraciones hechas sobre la frecuencia del Alzheimer y sus características de presentación entre las entidades neurológicas con especificación por género resulta importante insistir que por tendencias demográficas y estilos de vida de la población esta entidad está en una tendencia creciente al punto de entrar a ser no solo una patología neurológica de adultos mayores sino una de las mas importantes enfermedades cronicodegenerativas de la población por lo que es vital que se piense en planes de prevención que cambien hábitos nutricionales y de actividad física en adolescentes y adultos jóvenes para regular metabolismos de lípidos, y preparar las respuestas de acciones asistenciales integrales y pertinentes al contexto de la entidad para cuidar debidamente la calidad de vida y la dignidad de las personas en un proceso que es inexorablemente degenerativo no solo a nivel cognitivo sino físico por los cambios en las capacidades de planificar y desarrollar actividades de movimiento humano coherentes a contextos de desempeño.

Las limitaciones de este estudio corresponden a las de su tipo metodológico en el carácter transversal donde no se puede establecer elementos de causalidad, situación que no era de interés para el trabajo pues su centro de concentración era establecer la preva- lencia de la enfermedad de Alzheimer entre las entidades neurológicas vistas en Fisioterapia con sus características de presentación por género para tener un panorama descriptivo de estos ámbitos, y en cuanto al empleo del instrumento para la recolección de la información representado en los registros estadísticos mensuales de atención de pacientes en los sitios de Práctica del Programa de Fisioterapia de la Universidad Manuela Beltrán podría caber el hecho de un subregistro en la frecuencia de los pacientes con problemas neurológicos y particularmente con casos de Alzheimer lo que se reduce si se tiene en cuenta que estos registros son objeto de estricta verificación y control diario en las acciones asistenciales cumplidas por los docentes de práctica encargados de cada uno de los sitios aumentando la fiabilidad de las situaciones capturadas en ellos.

Se descarta elementos de sesgos de selección pues el investigador no escogió los sujetos con Alzheimer, ni ellos mismos pudieron influir en que ingresaran a formar parte de los datos del trabajo para el cálculo de las prevalencias y la magnitud encontrada en ellas, pues los datos de la frecuencia de la entidad provienen de los pacientes que por el diagnóstico de Alzheimer fueron atendidos y registrados en los servicios de Fisioterapia donde habían prácticas de la Universidad Manuela Beltrán en el año 2012, que fueron revisados con posterioridad a su diligenciamiento por el investigador.

Finalmente se descarta un sesgo de clasificación ya que los diagnósticos de Alzheimer y las patologías neurológicas de los pacientes fueron tomados de los registros estadísticos asistenciales teniendo en cuenta el diagnóstico por el cual médicamente se remitieron para el establecimiento de intervenciones terapéuticas por lo que ni las personas que desarrollaron la atención fisioterapéutica, ni el investigador tuvieron que ver en la determinación y especificación nominal de diagnóstico en ninguno de los pacientes que ingresaron en los registros estadísticos mensuales de atención.

En conclusión la enfermedad de Alzheimer tiene una prevalencia importante dentro de las entidades neurológicas, y muestra un predominio en su presentación en las mujeres. 


\section{REFERENCIAS}

Arai, H., Ouchi, Y., Yokode, M., Ito, H., Uematsu, H. et al (2012). Toward the realization of a better aged society: messages from gerontology and geriatrics. Geriatr Gerontolint 12 (1), 16-22

Boxer, A.L., Gold, M., Huey, E., Gao, F.B., Burton, E.A., et al (2013) Frontotemporal degeneration the next therapeutic frontier: molecules and animal models for frontotemporal degeneration drug development Alzheimers Dement 9 (2), 176-188

Chan, K.Y., Wang, W., Wu, J.J., Liu, L., Theodoratou, E., et al (2013) Epidemiology of Alzheimer's disease and other forms of dementia in China, 1990-2010: a systematic review and analysis Lancet 381 (9882), $2016-2023$

Franceschi, S. \& Wild, C.P. (2013) Meeting the global demands of epidemiologic transition - the indispensable role of cancer prevention. Mol Oncol $7(1), 1-13$

Gillette-Guyonnet, S., Secher, M. \&Vellas, B. (2013). Nutrition and neurodegeneration: epidemiological evidence and challenges for future research Br J ClinPharmacol 75 (3), $738-755$

Harrison J. (2013) Cognitive approaches to early Alzheimer's disease diagnosis Med Clin North Am 97 (3), 425- 438

Head E. (2013) A canine model of human aging and Alzheimer's disease BiochimBiophys Acta, 1832 (9), 1834-1839

Hernandez-Avila, M., Garrido-Latorre, F., \&Lopez-Moreno, S. (2000) Diseño de estudios epidemiológicos Salud Publica Mex, 42 (2), 144-154

Hernández B, \& Velasco-Mondragon, H. (2000) Encuestas transversales Salud Publica Mex 42 (5), 447-455

Jagust W. (2013) Vulnerable neural systems and and the borderland of brain aging and neurodegeneration Neuron 77 (2), 219-234

Keating, N. \&Gaudet, N. (2012) Quality of life of persons with dementia J Nutr Health Aging 16 (5), $454-456$

Letts, L., Edwards, M, Berenyi J, Moros K, O’ Neill C et al (2011) Using occupations to improve quality of life, health and wellness and client caregiver satisfaction for people with Alzheimer's disease and related dementias Am J OccupTher 65 (5), 497-504

Kern, A. \&Behl, C. (2009) The unsolved relationship of brain aging and late-on set Alzheimer disease. Biochim Biophys Acta 1790 (10), 1124-1132

Prevalencia de la Enfermedad de Alzeimer en Fisioterapia

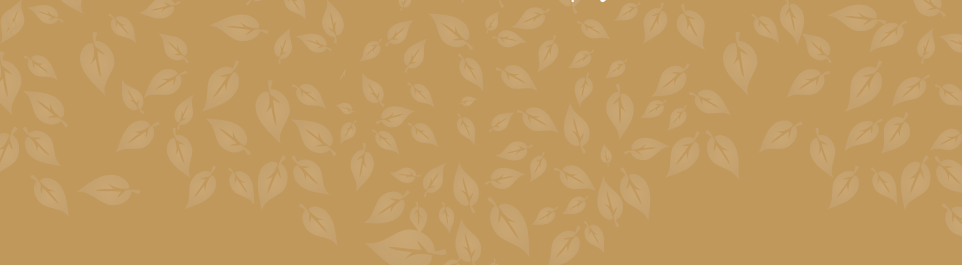

Loeser, R.F. (2010) Age-related changes in the musculoskeletal system and the development of osteoarthritis ClinGeriatr Med 26 (3), 371-386

Manderson, L. \&Naemiratch, B. (2010). From Jollibe to BeeBee: "life style" and chronic illness in Southeast Asia. Asia Pac J Public Health 22 (3), $117-124$

Miranda, J.J., Kinra, S., Casas, J.P., Davey Smith, G. \&Ebrahim, S. (2008) Non-comunicable diseases in low- and middle-income countries: context, determinants and health policy Trop Med Int Health 13 (10), $1225-1234$

Reitz, C. (2013) Dyslipidemia and the risk of Alzheimer's disease CurrAtheroscler Rep 15 (3), 307

Roman, G. \&Pascual, B. (2012) Contribution of neuroimaging to the diagnosis of Alzheimer's disease and vascular dementia Arch Med Res 43 $(8), 671-676$

Schapira, A.H. (2013) Recent developments in biomarkers in Parkinson disease Curr Opin Neurol, 26 (4), 395-400

Solfrizzi, V., Panza, F., Frisardi, V., Seripa, D., Logroscino, G. et al (2011) Diet and Alzheimer's disease risk factors or prevention: the current evidence Expert Rev Neurother 11 (5), 677-708

Solfrizzi, V., Frisardi, V., Seripa, D., Logroscino, G., Imbimbo, B.P. et al (2011) Mediterrean diet in predementia and dementia syndromes Curr Alzheimer Res 8 (5), $520-542$

Thal, D.R., Del Tredici, K., \&Braak, H. (2004) Neurodegeneration in normal brain aging and disease Sci Aging Knowledge Environ (23) , 26-30

Unger F. (2012) Health is wealth: considerations to european healthcare Prilozi 33 (1), 9-14

Uzun, Z., Kozumplik, O. \&Folnegovic-Smalc, V. (2011) Alzheimer's dementia: current data review Coll Antropol 35 (4), 1333 - 1337

VanWijngaarden, P. \& Franklin, R.J. (2013) Ageing stem and progenitor cells: implications for rejuvenation of the central nervous system $D e-$ velopment 140 (12), $2562-2575$

Vest, R.S. \& Pike, C.J. (2013) Gender, sex steroid hormones and Alzheimer's disease Horm Behav 63 (2), $301-307$

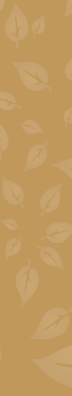
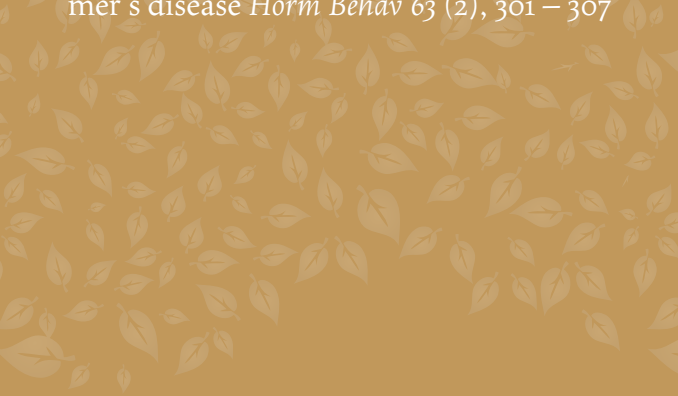